\title{
Erratum: Entanglement by Path Identity [Phys. Rev. Lett. 118, 080401 (2017)]
}

\section{Mario Krenn, Armin Hochrainer, Mayukh Lahiri, and Anton Zeilinger}

(Received 25 May 2017; published 23 June 2017)

DOI: 10.1103/PhysRevLett.118.259902

The statement that the experimental setup shown in Fig. 2(a) in Chap. III of the Supplemental Material creates a fivedimensional six-photon GHZ state is incorrect, as pointed out to us by Xuemei Gu. Likewise, the generalization of that statement in the main text for $n \geq 5$ photons with $d \geq 3$ dimensions is also incorrect. The reason is that more terms than just those of a GHZ state appear in the quantum superposition of the state realized by the setup in that Supplemental Material Fig. 2(a) and its generalizations. For example, the experimental setup for $n=6$ photons with $d=3$ dimensions produces the state $|\psi\rangle=1 / 2(|0,0,0,0,0,0\rangle+|1,1,1,1,1,1\rangle+|2,2,2,2,2,2\rangle+|1,2,1,2,0,0\rangle)$, where the first three terms are those of the GHZ state and the last term is the additional term. Detailed analysis of these additional terms have led to a new surprising link between quantum experiments and graph theory, which two of us present together with Xuemei Gu in Ref. [1].

All other results of the Letter remain valid. Particularly, all specific examples besides the one mentioned above are correct.

[1] M. Krenn, X. Gu, and A. Zeilinger, Quantum experiments and graphs: Multiparty states as coherent superpositions of perfect matchings, arXiv:1705.06646. 